\title{
Autonomous vehicles implementation in Lebanon: Opportunities, challenges, and ethical decisions
}

\author{
Elie Otayek ${ }^{1,{ }^{*}}$,Rania Wehbe ${ }^{1}$, and Zaher Massaad ${ }^{1}$ \\ ${ }^{1}$ Civil Engineering Department, Holy Spirit University of Kaslik - Usek, Lebanon
}

\begin{abstract}
The Autonomous Vehicle (AV) technology allows people to travel without intervening. The quick progress in this technology and the efforts of manufacturers companies to reach fully Autonomous Vehicles (AVs) using artificial intelligent, forecast a bright future. Researches have estimated that on average, one passenger in a vehicle traveling one kilometer in Lebanon costs about $\$ 48$, including externality components of crashes, travel time, congestion and pollution. Thus, the main purpose of this research is to highlight the opportunities of implementing AVs in Lebanon, such as reduction in traffic accidents and congestion, improvement of accessibility, human health, economic and social life. Moreover, this paper highlight the barriers standing in front of implementing this technology, like the missing of Intelligent Transportation System (ITS) and the funds needed for its execution, and gives some policy recommendations. Finally, the authors present their statistics conducted in Lebanon about ethical decisions that AVs should take particularly in case of a crash in different scenarios through the participants' responses.
\end{abstract}

\section{Introduction}

Lebanon is facing the challenge to reduce the number of accidents. According to the Internal Security Forces numbers, car accidents dropped by 20,62 \% in 2016 compared to 2015 . This decline was caused by the spread of awareness in Lebanese society through the publications about safety of the Non-Governmental Organization's NGO (KunHadi, Yasa, etc.), and the safety instructions by the Traffic Management Center (TMC) of Lebanon [1]. Despite this significant drop, the number of accidents in Lebanon remains high comparing to other countries. It is worth mentioning that approximately more than $90 \%$ of accidents are due to driver's faults [2].

The AV technology or self-driving vehicle can be the solution to lower the number of accidents and injuries since it can lead itself without human control, where computers software take over the art of driving. Moreover, many trial was completed by manufacturers to investigate their AVs, for example Tesla company have projected more than 210 million driving Kilometers from 2015 [3]. This kind of vehicle can solve many issues facing Lebanon like road safety problems, congestion, time delay, health and environmental impact. In addition, it increases the accessibility, social and economic development and eventually improving land use. 


\section{Opportunities and Benefits}

Manufacturers and technology companies agree on the advantages offered by AVs, whether in terms of safety, environmental and economic. Lebanon, a developing country, is facing with years an increase in the number of crashes, a high rate of pollution and a fall back in economic. What is the ability of autonomous vehicle to improve these standards?

\subsection{Road Safety}

The main reasons of traffic accidents in Lebanon (2011) are the excessive speed (22\%) and humans' inattention (21\%) [4]. Moreover, traffic accidents in Lebanon were expected to cost the national economy about $1.5 \%$ of its Gross Domestic Product (GDP) [4]. The autonomous vehicle is programmed to avoid accidents with the use of sensors. The implementation of $\mathrm{AVs}$ can be the solution to reduce the number of crashes, since the passenger is not responsible to control completely the car in a partially AV. The manufacturers and software companies are responsible to build safe vehicles that can really prevent any risk.

\subsection{Traffic Flow}

Congestion in Lebanon is one of the main problem facing Lebanon. Actually, the cost of road congestion is estimated around $\$ 2$ billion per year in Lebanon [5]. In addition, congestion affect the people moods and influence their health. The application of fully AVs will reduce the number of cars since many passengers can share one car.

\subsection{Accessibility}

AVs may afford mobility for children, young, elderly and disable people. The mobility of disable people can lessen the social isolation and a smooth access to important services.

Moreover, Lebanon is suffering from parking problem, and the idea of using new lots for parking spaces is not anymore the solution. The self-driving cars can prevent parking problems especially that vehicle can pick a person up and likewise drop them off, letting the car to drive multiple people to several locations

\subsection{Fuel Economic}

The main cause of pollution is due to vehicle emissions. The fossil fuel combustion and the emissions of pollutants cause damage to human health, agriculture, environments, and lead to global climate conversion.

The cost of environmental impact in Lebanon was estimated to be close to USD 485 million per year, and it represents a high percentage of GDP around 2.9\% [6].

Vehicles emissions depends on many factors such as congestion and travel time. AV will decrease the congestion problem, with less stop and go traffic, with fewer vehicles and smooth travel. Furthermore, AV will reject the successive changing in speed and continuous braking, then it can lead to fuel efficiency.

\subsection{Economic Improvement}

Lebanon is facing a retreat in economic growth in the last few years to reach a $2 \%$ GDP annual growth rate in 2016 [7]. The AV technology would make a huge influence on economic upgrading, especially on the land shipping industries. Trucks are usually used to 
move freights on land, and most of time the drivers take multiple hours and days to transport goods to its destinations [8]. The use of autonomous trucks would increase the productivity of the industries and trucks companies by saving money.

The influence of AVs is not limited to trucks facilities, all the restaurants and stores will benefit from the smooth delivery option without a paid employee. For example, Domino's Pizza in U.S. is using a fully autonomous vehicle to deliver pizza and it is getting good feedbacks. Moreover, Lebanese people do not use frequently the taxi and public transport because of driver's behavior and their non-adoption of laws. The autonomous bus or taxi will be able to follow the rules and act properly.

\subsection{Time Saving}

Lebanese people lose time on roads, to reach their destination. They are usually late to their work, meetings and activities. AV will force the passengers to reach their destinations on time more than before and they will have more time for leisure activities.

$\mathrm{AV}$ can also reduce the population in busy cities like Beirut. Urban population in Lebanon cover around $88 \%$ of total population in 2017 [9], a percentage of Lebanese who work or study in cities live there. The self-driving vehicles can push the people to live far due to the transportation facilities.

\section{Barriers and Challenges}

AVs present many opportunities and it is progressing faster than regulations. But, many barriers prevent the implementation of this technology in Lebanon as illustrated in figure 1. In this section we focused on the barriers facing the implementation of AVs in Lebanon.

\subsection{Infrastructure Barriers}

The autonomous vehicle depends on ITS system where the communication between vehicles $\mathrm{V} 2 \mathrm{~V}$ and vehicle to infrastructure V2I is valid. As a first step, Lebanon should improve the infrastructure system by switching to a smart city.

\subsection{Legal Barriers}

Lebanon and most Arab countries signed international agreements and conventions, including Geneva Conventions in 1949 and Vienna in 1968. The Article 8 and 13 of the Vienna Convention put emphasis on the importance of having a driver responsible for each vehicle on the road. In other hand, the Geneva Convention allows manufacturer companies to control the vehicle instead of a driver. Lebanon are not able to implement this technology until the modification of those articles. A suggestion has been submitted for Vienna Convention to modify the articles in a way to force the driver to control the car when he should do, not all the time [10]

\subsection{Rules Proposal}

The state should start by placing clear rules for the test and implementation stage of the AV. It is very important to discriminate between the regulations in case of a partially and a fully autonomous vehicle. In case of a fully AV accident, who should be responsible the owner, the software engineer or the manufacturer company? According to the survey conducted in 
Lebanon with 380 participants, $22 \%$ reflect that the owner is responsible, $45 \%$ holds the responsibility on the software engineer and $33 \%$ on the manufacturer company.

\subsection{Kilometres' of Testing}

The success of AV outside Lebanon is not guaranteed on Lebanon roads. At that moment, those devices should be tested in Lebanon before launching them on roads.

\subsection{Public Acceptance}

This barrier could be solved easily when the regulations are clear in Lebanon about the use of AVs. Jonat Harris a creative writer reflect that persuade people about the importance of $\mathrm{AV}$ in improving road safety, can accelerate the implementation phase of this technology [11]. People should feel protected and safe to trust the machine. About $75 \%$ of the Lebanese participants believe that AV can reduce crashes. However, people cannot trust any machine without knowing all its features, rules and opportunities.

\subsection{Security}

Policymakers and future autonomous vehicle drivers worry about electronic hacks. Computer hackers may aim AVs and ITS to produce crashes and traffic troubles.

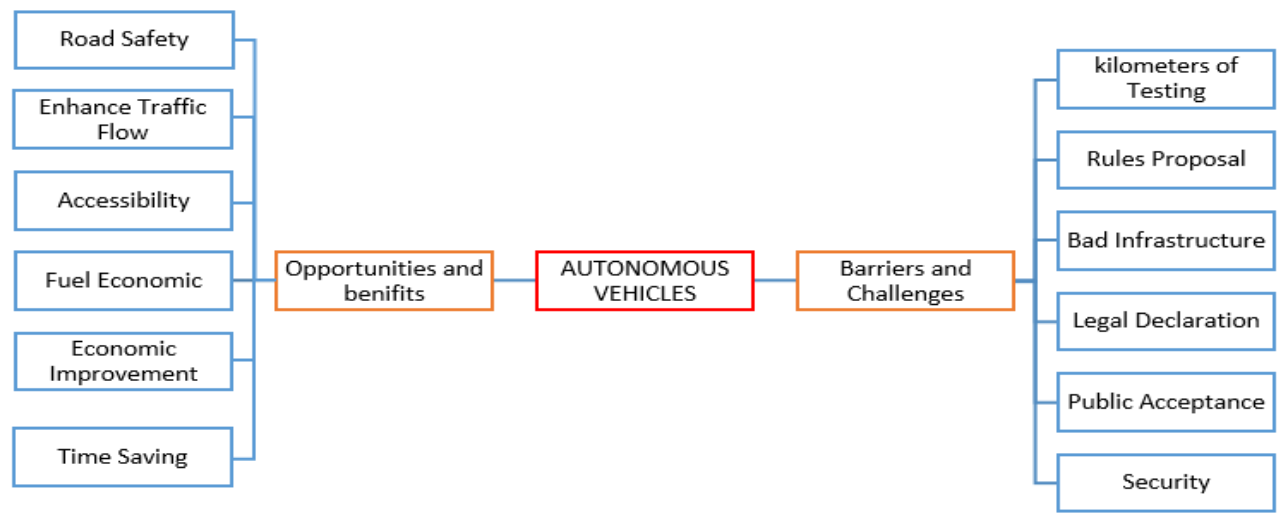

Fig. 1. Autonomous Vehicles opportunities and challenges in Lebanon

\section{Ethical Difficulties}

AV has progressed rapidly in improving machine knowledge, sensing, and manufacturing. The perfection of machine learning is based on artificial intelligent that comes with many forms and many features. This program can solve tasks that need the intelligence of humans. The main problem standing in front of launching the autonomous vehicles on road is their ability to make ethical decisions in case of a crash.

A survey was conducted in Lebanon to know the ethical behavior of 380 passengers by asking some hypothetical examples. According to the survey, around $54 \%$ of the participants believe that AV can be used in Lebanon but approximately $92 \%$ reflect that our infrastructure system in Lebanon is not qualified for the use of AV. Some of peoples' suggestions to improve Lebanon infrastructure are: (A) Better roads should be designed in order to allow 
for higher flow facilities such as clear pedestrian walk sides, high performance intersections, proper drainage system, and smaller bumps on roads that are not harmful to cars; (B) Maintenance of roads; and (C) Move to smart infrastructures before using a driverless car then the use of ITS.

About $75 \%$ of the participants declare that they will buy an autonomous vehicle if it becomes legal in Lebanon. Furthermore, approximately $87 \%$ prefer vehicle partially controlled by the driver for several reasons: (A) the human brain can take better decisions, nothing can be done totally by a machine; (B) avoid total domination of electronics; and (C) intervention of the driver in case of technical or software damage. In addition, $13 \%$ of participants prefer vehicle fully controlled by technology, they believe that (A) technology will be very powerful in the upcoming years; (B) it would follow regulations; and (C) it would be more comfortable and safe.

The authors proposed several scenarios where no escape from the collision so they can expect the human brain reaction in similar situations when driving. The first question was about a sudden brake failure in the car, what should the self-driving car do, save five pedestrians crossing the road or the five passengers in the car? Around 57\% desired to save their lives and hit the passenger. Nevertheless, when the same question was asked with a small modification by considering one passenger and two pedestrians, $80 \%$ preferred to kill themselves to save two lives. In the case of a dog passing suddenly the road, $79 \%$ decided to murder the dog. From those three situation, it can be concluded that around $57 \%$ of the participants aims to protect the passengers and $79 \%$ aims to save more lives.

In order to recognize how important for the driver to follow the law and if he or she differentiates between social values, we put the participants in a situation where they should choose to hit between one criminal man who is crossing the road on green signal and one girl crossing the road on red signal. About $67 \%$ decides to hit the girl crossing the road on red signal. When they were asked about two options to hit an elderly man or a young boy, $86 \%$ preferred to hit the elderly man and to save the young boy.

The target of this survey is to have a moral knowledge about the factors and their level of importance in driver's judgement while driving.

\section{Conclusion}

Lebanon, has a cheap public transport network, and need an evolution in its infrastructure. The AVs offer the opportunities to somewhat eliminate the transportation facilities weakness. This technology can improve road safety and traffic flow by dropping congestion problems and fuel emissions, bring mobility for children, elderly and disable people subsequently improve the social life. In addition, it can develop the economic sector, condense the need of parking spaces and save time for passengers.

However, the challenge is to implement this technology in Lebanon, by braking the barriers standing in front of adopting AVs. The weakness of Lebanon infrastructure is a major obstacle, because this technology depends on the communication between V2V and V2I, and the absence of clear regulations and rights for the use of autonomous do not allow people to trust this technology. In addition, the researches and testing for the self-driving cars on Lebanon roads is still not available.

To follow the quick growth of technology, the responsibility falls first on the manufacturers and technology companies, policymakers and the public.

To summarize, the future works to investigate the autonomous vehicle very shortly in Lebanon is to set clear regulations for fault in advance, based on past scenarios, ethical decisions and mathematical model. 


\section{References}

1. Najib. (2016, December 30). 2016 Statistics: Car Accidents Down by 20\%. Retrieved from BlogBaladi: http://blogbaladi.com/2016-statistics-car-accidentsdown-by-20/

2. National Highway Traffic Safety Administration. (2008). National Motor Vehicle Crash Causation Survey. Washington D.C.: U.S.: Department of Transportation, Report DOT HS 811059.

3. Bjornstad, E. (2016, August 23). Self-Driving Car Accidents - Tesla and Google . Retrieved from Bell Performance: https://www.bellperformance.com/blog/selfdriving-car-accidents-tesla-and-google

4. Choueiri, E. M., Choueiri, G. M., \& Choueiri, B. M. (2012). An Overview About Road Safety In Lebanon. Ideas.

5. Marsi, F. (2017). New transport plan aims to ease traffic. Pressreader.

6. El Khoury, N., Aboujaoude, L. K., \& Kadehjian, K. (2016). Road Transport Sector and Air Pollution Case of Lebanon 2016. Lebanon.

7. Banque du Liban. (2018, February 25). Lebanon GDP Annual Growth Rate. Retrieved from Trading Economics: https://tradingeconomics.com/lebanon/gdpgrowth-annual

8. Fagnant, D. J., \& Kockelman, K. (2015). Preparing a Nation for Autonomous Vehicles: Opportunities, Barriers and Policy Recommendations for Capitalizing on Self-Driving Vehicles. Transportation Research Part A 77, 167-181.

9. CIA World Factbook. (2018, January 20). Lebanon Demographics Profile 2018. Retrieved from Index Mundi: https://www.indexmundi.com/lebanon/demographics_profile.html

10. Moutran, M. (2017). Self-driving Vehicles Legal and Technical Challenges. Lebanon: Printing \& Publishing Company.

11. Harris, J. (2017, November 8). Autonomous Vehicles to Reduce Road Fatalities by More than 50 Percent. Retrieved from Sanvada: https://sanvada.com/2017/11/08/autonomous-vehicles-to-reduce-road-fatalitiesby-more-than-50-percent/ 